\title{
Time-dependent thermoelectric transport in nanosystems: reflectionless Luttinger field approach
}

\author{
Č. Lozej \\ CAMTP - Center for Applied Mathematics and Theoretical Physics, \\ University of Maribor, Mladinska 3, Maribor, Slovenia \\ T. Rejec* \\ Faculty for Mathematics and Physics, University of Ljubljana, Jadranska 19, Ljubljana, Slovenia and \\ Jožef Stefan Institute, Jamova 39, Ljubljana, Slovenia
}

(Dated: July 13, 2018)

\begin{abstract}
Recently the Luttinger field approach was proposed by Eich et al. in Phys. Rev B 90, 115116 (2014) as a way to simulate switching on a temperature gradient across a nanoscale device initially in thermal equilibrium. The time dependent particle and heat currents can then be calculated by propagating the initial equilibrium state of the system in time. Unfortunately applying a uniform Luttinger field in the whole of a lead causes a discrepancy between steady state currents in the long time limit and those predicted by the Landauer-Büttiker formulas as well as artefacts at short times. Here we propose a modified approach where the Luttinger field gradually reaches its final value across a transition region. If the length of the transition region is sufficient, the electrons move through without reflecting. In this way the correct energy distribution of electrons originating from such a lead, corresponding to the new temperature, is established in the scattering region. Our approach is tested on a single quantum dot and a parallel double quantum dot system.
\end{abstract}

\section{INTRODUCTION}

The gradual improvement of nanotechnology has long been an inspiration for the development of a quantum theory of transport at the nanoscale. While the steady-state particle and heat currents through a nanodevice can successfully be calculated via the LandauerBüttiker (LB) formalism ${ }^{1-3}$, there is a growing interest in developing methods for time-dependent transport calculations $^{4,5}$. Some recent attention has been given to particle and heat transport due to temperature gradients, with possible practical applications in thermoelectric energy conversion (see Ref. [6] and references therein). This, however, raises some fundamental questions about the microscopic aspects of temperature when the system is out of equilibrium. In standard thermodynamics we define temperature as a statistical parameter of macroscopic parts of a system in local quasiequilibrium conditions. The temperature is thus not explicitly represented in the Hamiltonian of the system studied. If we wish to model time dependent temperature gradients in order to study thermal transport at the nanoscale we need a way of coupling the temperature changes to the electron dynamics.

A solution for this problem was proposed by Luttinger ${ }^{7}$. To simulate the effects of a temperature gradient he proposed the introduction of a scalar potential field, which couples to the electronic energy density. This approach has been applied in the calculation of the linear response in macroscopic systems ${ }^{8}$ and has recently been adopted for the inclusion of thermoelectric effects in a general-purpose density-functional theory ${ }^{9,10}$. It has been further shown that the the steady-state electron and heat currents when switching on the Luttinger field coincide with those derived from the Landauer-Büttiker
(LB) formalism in the linear transport regime, but show marked differences in the nonlinear regime ${ }^{11}$. Alternatively the same linear regime steady-state properties due to a temperature gradient may be obtained by the introduction of a thermal vector potential ${ }^{12,13}$. Another completely different approach involves replacing the temperature gradient with controllable external blackbody radiation ${ }^{14}$.

In the well received work ${ }^{11}$ Eich et al. proposed using the Luttinger field as a way to simulate switching on a temperature gradient between the leads of a nanoscale junction which was initially in thermal equilibrium. Switching on the Luttinger field drives the system out of the equilibrium causing particle and heat transport to occur. While in the linear response regime the steady state currents in the long time limit agree with those calculated from the LB formulae, in the nonlinear regime this approach predicts steady state currents which are too small. In Ref. [15] the authors extended their work by calculating the transient response and found that immediately after the Luttinger field is switched on, the currents flow back into the lead where the temperature rise is simulated. They change the direction only somewhat later and then rise up to their steady state value. Similarly, in Ref. [16] a discontinuity in currents was found when the temperature gradient is applied. We traced the cause of both the discrepancy of the steady state currents and the initial oscillation to the fact that the Luttinger field changes the width of the energy bands abruptly at the interface between the lead and the scattering region. Additional scattering of the electrons on the resulting field step is responsible for the discrepancies discussed above. To rid ourselves of those artifacts we propose to introduce a transition region where the Luttinger field changes gradually from zero at the interface to the value 
corresponding to the final temperature in the lead. If the transition region is long enough, the Luttinger field is reflectionless and no additional scattering occurs, bringing the steady state currents up to the LB value and removing the artifacts in the transient currents at short times.

In Sec. II we describe the system we are studying, its Hamiltonian and write down the expressions for the particle and heat currents through the system. We then discuss how to impose a reflectionless Luttinger field in one of the leads to simulate the increase of the temperature there and compare the state the lead evolves to to that in the case of a uniform Luttinger field. In Sec. III we present the numerical results for the time evolution of the electron and heat currents for the case of a single quantum dot device and a parallel double quantum dot device. The results and main conclusions are summarized in Sec. IV. The details of the calculations are presented in Appendices A and B.

\section{MODEL}

We consider a nanoscopic transport setup consisting of two conducting leads each coupled to its own thermal reservoir and joined by way of a general central scattering region. For the sake of simplicity we consider spinless fermions and model the leads as one-dimensional tight binding chains. This can easily be generalized to include spin and general translationally invariant leads. The lattice constant is set to $a=1$. The Hamiltonian of the model system may be written as

$$
H=H_{C}+\sum_{\alpha} H_{\alpha}+\sum_{\alpha}\left(H_{C \alpha}+H_{\alpha C}\right),
$$

where $H_{C}$ is a general tight-binding Hamiltonian describing the scattering region, $H_{\alpha}$ is the lead Hamiltonian with the index $\alpha \in\{L, R\}$ denoting the left and right lead, respectively, and $H_{\alpha C}$ and $H_{C \alpha}=H_{\alpha C}^{\dagger}$ describe the couplings of the leads to the scattering region. The Hamiltonian of the scattering region may be written as

$$
H_{C}=\sum_{i i^{\prime}} H_{i i^{\prime}} d_{i}^{\dagger} d_{i^{\prime}}
$$

where $d_{i}^{\dagger}$ and $d_{i}$ are the fermionic creation and annihilation operators on site $i$ of the scattering region. The leads are modeled as semi-infinite one-dimensional chains

$$
H_{\alpha}=\sum_{j=0}^{\infty}-\gamma c_{\alpha j+1}^{\dagger} c_{\alpha j}+\text { h.c. }
$$

where $c_{\alpha j}^{\dagger}$ and $c_{\alpha j}$ are the fermionic creation and annihilation operators on site $j$ of lead $\alpha$ and $\gamma$ is the hopping parameter. The dispersion relation is given by

$$
\varepsilon(k)=-2 \gamma \cos k,
$$

where $\varepsilon$ is the energy of the single-electron eigenstate and $k$ is the wavevector. We set $\hbar \rightarrow 1$ and measure all energies in terms of $\gamma$, and times in terms of inverse energy. The coupling terms are written as

$$
H_{\mathrm{C} \alpha}=\sum_{i}-\gamma_{i \alpha} d_{i}^{\dagger} c_{\alpha 0}
$$

where $c_{\alpha 0}$ is the annihilation operator of the first site of lead $\alpha$.

We are interested in the particle and heat currents into the right lead after the electrons cross the scattering region. The electron current is calculated as

$$
I=\frac{d}{d t}\left\langle N_{R}\right\rangle=i\left\langle\left[H, N_{R}\right]\right\rangle=2 \sum_{i} \gamma_{i R} \operatorname{Im}\left\langle d_{i}^{\dagger} c_{R 0}\right\rangle,
$$

where $N_{R}$ is the number operator counting electrons in the right lead. The angled bracket denotes the thermal expectation value. The state occupation in each lead is given by the Fermi-Dirac distribution

$$
f_{\alpha}(\varepsilon)=\frac{1}{\exp \left(\frac{\varepsilon-\mu_{\alpha}}{T_{\alpha}}\right)+1}
$$

where $\mu_{\alpha}$ is the chemical potential and $T_{\alpha}$ is the temperature of lead $\alpha$ given in terms of energy units with the Boltzmann constant set to $k_{B} \rightarrow 1$. We set the chemical potentials to $\mu_{L}=\mu_{R}=0$. Similarly, the heat current is calculated as

$$
I_{Q}=\frac{d}{d t}\left\langle H_{R}\right\rangle=i\left\langle\left[H, H_{R}\right]\right\rangle=2 \gamma \sum_{i} \gamma_{i R} \operatorname{Im}\left\langle d_{i}^{\dagger} c_{R 1}\right\rangle .
$$

\section{A. Luttinger field}

Initially the system is considered in equilibrium conditions at temperature $T_{0}$ in both leads. At $t=0$ the temperatures in the leads are instantaneously changed to new values $T_{\alpha}$. As described in Ref. [11] we may simulate this by turning on a suitable Luttinger field in the leads at $t=0$. The Luttinger field approach enables us to simulate the temperature change in the lead Hamiltonians instead of changing the occupation of the single-electron states. The lead Hamiltonians become time-dependent. We select a number of initial single-electron scattering states with wavevectors on a uniform mesh in each of the leads. The states are propagated in time by the timedependent Hamiltonian using the numerical scheme described in Appendix A. The currents into the right lead, Eqs. (2) and (3), are calculated using the initial occupations, as explained above.

Next, we shall describe the the time-dependent lead Hamiltonians. For the sake of simplicity we will consider a temperature change in only the left lead. Let us assume the temperature in the left lead is raised abruptly 
to $T_{L}>T_{0}$. The Hamiltonian of the left lead thus acquires a simple time dependence in the form of rescaling the hopping parameter at the $t=0$ :

$$
H_{L}(t>0)=\sum_{j=0}^{\infty}-\gamma_{j+1, j} c_{L j+1}^{\dagger} c_{L j}+\text { h.c. }
$$

Instead of a uniform Luttinger field in the whole of the lead we may choose to introduce a transition region where the field, being zero at the junction of the lead and the scattering region, reaches its final value in the bulk of the lead gradually. We chose the following functional form for the spatial dependence of the hopping parameter

$$
\gamma_{j+1, j}= \begin{cases}\gamma\left(1+\left(\frac{T_{L}}{T_{0}}-1\right) \sin ^{2} \frac{\pi}{2} \frac{2 j+1}{2 j_{0}}\right) ; & j<j_{0}, \\ \gamma \frac{T_{L}}{T_{0}} ; & j \geq j_{0},\end{cases}
$$

where $j_{0}$ is the number of lattice sites forming the transition region. If $j_{0}=0$, (discontinuous limit), the hopping parameter is uniformly equal to $\gamma \frac{T_{L}}{T_{0}}$ in the whole of the left lead. In contrast, if $j_{0}$ is large, the hopping parameter changes smoothly (reflectionless limit). The two regimes are illustrated in Fig. 1.



b)
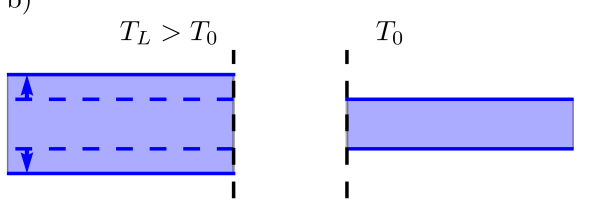

c)

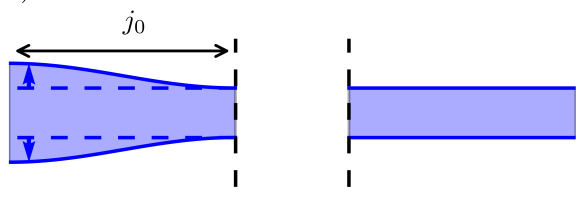

Figure 1. a) A schematic representation of a nanoscale device attached to leads. b) Energy bands in the leads before (dashed line) and after (solid line) a rise of temperature in the left lead in the discontinuous limit. c) The same in the reflectionless limit.

\section{B. Device with no scattering region}

Let us first consider the case of a device without a scattering region, consisting of only a lead coupled to thermal reservoirs at each end. In our framework this can be represented as a device where the left and right lead are coupled to each other directly, the scattering region thus containing no sites. The steady state electron current through the lead is calculated by the LB formula

$$
I=\int d \varepsilon\left(A_{L}^{(I)}(\varepsilon) f_{L}(\varepsilon)-A_{R}^{(I)}(\varepsilon) f_{R}(\varepsilon)\right)
$$

where $f_{L}(\varepsilon)$ and $f_{R}(\varepsilon)$ are the Fermi-Dirac distributions of electrons originating from left and right, respectively. Here, we introduced the current spectral functions

$$
A_{\alpha}^{(I)}(\varepsilon)=\frac{d k}{d \varepsilon} j_{\alpha}(k)= \begin{cases}1 ; & |\varepsilon|<2 \gamma \\ 0 ; & |\varepsilon|>2 \gamma\end{cases}
$$

where $j_{\alpha}(k)$ is the probability current corresponding to a single-electron eigenstate with wavevector $k, \alpha \in\{L, R\}$.

The current spectral functions can be generalized to the time dependent situation by evaluating the probability currents from the time-evolved single-electron states. We examine the effects of turning on the Luttinger field in the left lead on the current spectral functions for the current into the right lead. Initially, the hopping parameter is uniform across the whole chain of sites and set to $\gamma=1$. Then at $t=0$ the Luttinger field is switched on, i.e. the hopping parameters in the left lead are rescaled, as described in Subsection II A, by a factor of $\frac{T_{L}}{T_{0}}=2$. The initial temperature is set to $T_{0}=0.1$. Physically no electron scattering should occur in the junction of the two leads as one lead differs from the other only in its temperature. The current spectral functions in the initial equilibrium state as well as the final steady state should be uniform.

Fig. 2a) shows a color plot of the time dependence of $A_{L}^{(I)}(\varepsilon)$ for the case of the discontinuous transition of the energy band, $j_{0}=0$. The profiles of the current spectral function at chosen times are depicted in panel b) of the same Figure. Before the rescaling of the hopping parameter, the current spectral function is, indeed, distributed uniformly across the width of the energy band. When the hopping parameter is rescaled the distribution is stretched across the new width of the energy band. This is due to the fact that the dispersion relation used to convert the variable from the wave vector to the energy used in the plots is that of the rescaled energy band $\varepsilon\left(k_{L}\right)=-2 \frac{T_{L}}{T_{0}} \gamma \cos k_{L}$, where $k_{L}$ is the wavevector in the left lead. We see that the new distribution is markedly different from the uniform distribution the system should have evolved to. Panels c) and d) in Fig. 2 show the same quantities as panels a) and b) for $A_{R}^{(I)}(\varepsilon)$, respectively. This current spectral function is not stretched as the electrons originate from the right lead where the temperature is left unchanged. Nevertheless the long time distributions, are again different from the expected uniform distribution.

These discrepancies are due to the abrupt change in the electron energy band where the left and right leads meet. The hopping parameter changes discontinuously from $\gamma \frac{T_{L}}{T_{0}}$ in the left lead to $\gamma$ in the right lead. Because the energy band on the left is rescaled, the inequality 

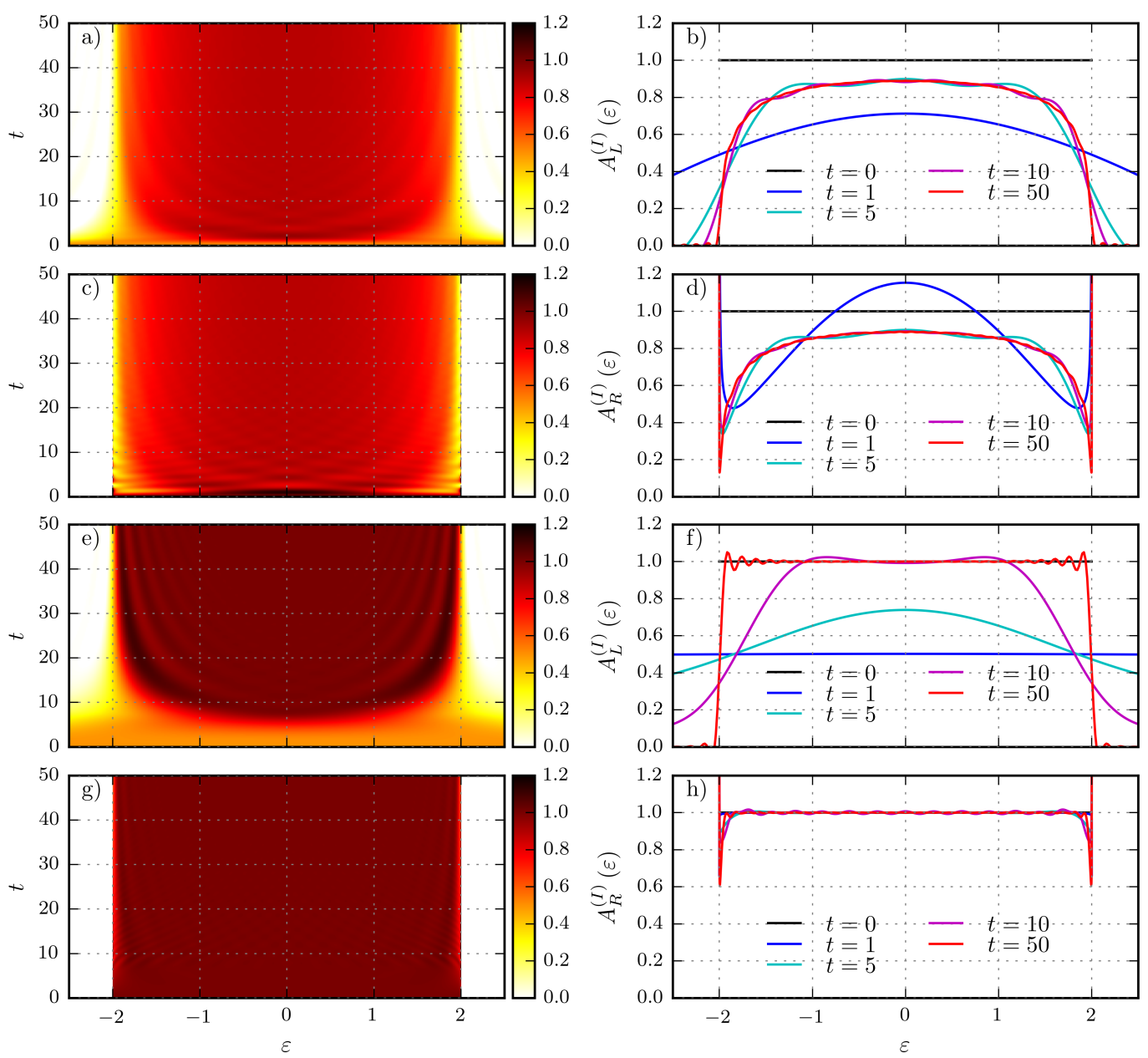

Figure 2. Current spectral functions for the current into the right lead in the device with no scattering region, a) for the left lead $A_{L}^{(I)}(\varepsilon)$ and c) for the right lead $A_{R}^{(I)}(\varepsilon)$ as a function of time in the discontinuous case, $j_{0}=0$. b) and d) show the current spectral functions at various times. e), f) show the same quantities as a), b) and g), h) the same as c), d) in the reflectionless limit, $j_{0}=20$. The temperatures are $T_{0}=0.1$ and $T_{L}=0.2$.

$|\varepsilon|>2 \gamma$ holds for certain single-electron states originating from the left. These states are reflected on the barrier formed by the discontinuous change in the energy band. Furthermore, scattering occurs even for the eigenstates whose eigenenergies lie within the original energy band $|\varepsilon|<2 \gamma$. The transmission probability is

$$
\mathcal{T}(\varepsilon)=\frac{4 \frac{T_{L}}{T_{0}} \gamma^{2} \sin k_{L} \sin k_{R}}{\left(\gamma \frac{T_{L}}{T_{0}}\right)^{2}+\gamma^{2}-2 \frac{T_{L}}{T_{0}} \gamma^{2} \cos \left(k_{L}+k_{R}\right)},
$$

where the wavevectors $k_{\alpha}$ are given by the local dispersion relations $\varepsilon=-2 \frac{T_{L}}{T_{0}} \gamma \cos k_{L}$ in the left lead and $\varepsilon=-2 \gamma \cos k_{R}$ in the right lead. Because of this additional scattering some of the electron current is lost as can most clearly be seen in panels b) and d) of Fig. 2 .

The above described artefacts of simulating a temperature change with Luttinger field may be amended by the introduction of a longer transition region. Panel e) of
Fig. 2 shows the same quantities as panel a) for a transition region of length $j_{0}=20$. Panel f) shows the profiles of $A_{L}^{(I)}(\varepsilon)$ at chosen times. As before the current spectral function is stretched when the hopping parameters are rescaled. In contrast to the discontinuous case, the current spectral function evolves back to the expected uniform distribution. Panels g) and h) show the same quantities as c) and d) in the case of the long transition region. We see that the current spectral functions for the electrons originating from the right side remain mostly unaffected by the rescaling of the energy band.

An analytical calculation of the single-electron eigenstates for the initial equilibrium and the final steady state of the system in the reflectionless limit is possible. The details of the derivation are given in Appendix B. A local dispersion relation

$$
\varepsilon=-2 \gamma_{j} \cos k_{j}
$$


holds for each site of the lead, where $\varepsilon$ is the eigenenergy, $k_{j}$ is the local wavevector and $\gamma_{j}$ is the hopping parameter near site $j$. The single-electron states with eigenenergies $|\varepsilon|>2 \gamma_{j}$ are reflected as was the case when the band transition was abrupt, but no scattering occurs for the single-electron states with eigenenergies $|\varepsilon|<2 \gamma$. These states are transmitted fully and thus no current is lost due to unwanted backscattering.

The transition region introduces a time delay in the current response of the system. The characteristic time $\tau$ needed to reach the stationary current spectral functions can be associated with the local group velocity of the electrons $v_{j}=2 \gamma_{j} \sin k_{j}$. The local group velocity is largest in the middle of the energy band and smallest on the edge of the band. The characteristic time $\tau$ needed to reach stationarity is the length of the transition region divided by the average velocity of the electrons. If we approximate $\gamma_{j} \approx \gamma=1$, we expect $\tau(\varepsilon)=\frac{j_{0}}{2 \sqrt{1-\left(\frac{\varepsilon}{2}\right)^{2}}}$. We can assess the characteristic time $\tau(\varepsilon)$ from the time dependence of the current spectral function shown in Fig. $2 \mathrm{e})$. The shape of the contour when the current spectral function reaches its uniform steady state is indeed the expected one. We estimate the characteristic time in the middle of the band to $\tau=j_{0} / 2=10$. We compare this to the cross section of $A_{L}^{(I)}(\varepsilon)$ at $t=10$ depicted in Fig. 2f). In the middle of the energy band the current spectral function is indeed close to the uniform steady state at this time.

We have thus shown that a straightforward approach using a uniform Luttinger field to rescale the hopping parameters causes additional scattering to occur at the discontinuity. This can be avoided by introducing a transition region of sufficient length where the hopping parameter is smoothly varied in order to reach the reflectionless limit. We will refer to this as the reflectionless Luttinger field approach.

\section{RESULTS}

Here, we present the numerical results for the timedependent electron and heat currents in two chosen transport setups. In both cases the setup is comprised of two one-dimensional leads connected via a central scattering region. In equilibrium the hopping parameter of the leads is set to $\gamma=1$. At time $t=0$, the energy band in the left lead is changed by switching on the Luttinger field, as described in Subsection II A. The scattering region is either a single site, representing a quantum dot or two sites representing a double quantum dot device. The Hamiltonian of the scattering region and the coupling to the leads in the single quantum dot case may be written as

$$
\begin{gathered}
H_{C}=\varepsilon_{0} d_{0}^{\dagger} d_{0}, \\
H_{C L}=-\gamma_{L} d_{0}^{\dagger} c_{L 0}, \\
H_{C R}=-\gamma_{R} d_{0}^{\dagger} c_{R 0},
\end{gathered}
$$

where $\varepsilon_{0}$ is the quantum dot energy. The couplings to the leads determine the resonance width $\Gamma=2 \gamma_{L}^{2} / \gamma+2 \gamma_{R}^{2} / \gamma$.

In the second device the scattering region consists of two quantum dots in a parallel configuration,

$$
\begin{gathered}
H_{C}=\varepsilon_{1} d_{1}^{\dagger} d_{1}+\varepsilon_{2} d_{2}^{\dagger} d_{2}, \\
H_{C L}=-\gamma_{L} d_{1}^{\dagger} c_{L 0}-\gamma_{L} d_{2}^{\dagger} c_{L 0}, \\
H_{C R}=-\gamma_{R} d_{1}^{\dagger} c_{R 0}-\gamma_{R} d_{2}^{\dagger} c_{R 0},
\end{gathered}
$$

where $\varepsilon_{1}$ and $\varepsilon_{2}$ are the energies of the first and the second quantum dot, respectively. The resonance width is calculated in the same way as in the single quantum dot case. There is no direct hopping between the two dots.

We calculated the currents into the right lead, Eqs. (2) and (3), by integrating over $k$ using an equidistant mesh with mesh spacing $2.5 \times 10^{-3}$.

\section{A. Single quantum dot device: dependency on the length of the transition region}

The length of the transition region in the left lead is an important parameter in our application of the Luttinger field approach. We have shown that a very short transition region may potentially lead to unphysical results. This is demonstrated for the case of the electron and heat currents from the single quantum dot device into the right lead in Fig. 3. Panel a) depicts the time dependencies of the electron currents, corresponding to an instantaneous change of the temperature in the ratio of $T_{L} / T_{0}=2$ for different lengths of the transition region. Panel b) shows the heat currents in the same cases. In order to test the validity of our numerical results, we compare the asymptotic values of the electron and heat currents with the LB steady state currents plotted with a dashed line. We see that for insufficiently long transition regions the asymptotic values of the electron and heat currents differ from the LB steady state currents. We also note that initially the currents may flow in the opposite direction of the steady-state currents. This was also observed in Ref. [15]. These effects are diminished when the transition region is lengthened, but a time delay $t_{0}$ in the current response occurs which is related to the travel time needed for the electrons to travel the distance of the transition region. The reflectionless limit is reached when the asymptotic currents start to coincide with the LB steady state currents. The further lengthening of the transition region changes only the initial time delay. This occurs at about $j_{0}=10$, as may be seen in Fig. 3 for both the electron and heat currents. For other values of $T_{L}$, the required length can be estimated as $j_{0}=10 \sqrt{\left(T_{L}-T_{0}\right) / T_{0}}$ for our shape of the transition region.

In Fig. 4 the time evolution of the current spectral functions in the quantum dot device is presented. Panels a), b), c) and d) depict the results for no transition region with $j_{0}=0$ and panels e), f), g) and h) depict the 



Figure 3. The time dependence of a) the electron current and b) the heat current from the quantum dot into the right lead calculated for different lengths of the transition region. The parameters are $\varepsilon_{0}=0.2, \Gamma=0.05, T_{0}=0.1, T_{L}=0.2$. The LB current is plotted with the black dashed line.

results in the reflectionless limit with $j_{0}=20$. Due to the resonant tunneling of electrons to and from the quantum dot, the current spectral function exhibits a sharp resonance at the quantum dot energy. When the Luttinger field is turned on, the current spectral function in the left lead is stretched across the new width of the energy band in the same way as seen in the case without a scattering region in Subsection IIB. In the single quantum dot device the resonance peak in the left lead is moved to $\frac{T_{L}}{T_{0}} \varepsilon_{0}$ at the start of the time evolution as seen in Fig. 4. The resonance then relaxes back to the initial form in the reflectionless limit, but not fully in the case of the discontinuous change of the hopping parameter (compare panel b) with panel f)). Furthermore, we note that the current spectral function in the right lead is left unchanged by the Luttinger field introduced on the left lead only in the reflectionless case (compare panel d) with panel h)).

\section{B. Single quantum dot device: dependency on quantum dot parameters}

Next, we present the numerical results for the timedependent electron and heat currents from the single quantum dot device into the right lead in the reflectionless limit, with $j_{0}=20$ for various values of parameters $\varepsilon_{0}$ and $\Gamma$. Figure 5 shows the time dependencies of the electron, panel a) and heat currents, panel b), corresponding to an instantaneous change of the temperature in the left lead in the ratio of $T_{L} / T_{0}=2$. In all presented cases both the the final electron as well as heat currents coincide with the LB steady state currents (not shown). The time scale of the current response is dictated by the resonance width $\Gamma$. This is shown in the insets of Fig. 5 . The initial time delay due to the long transition region was estimated to $t_{0}=7$ time units. If the currents are rescaled by the $\mathrm{LB}$ values and plotted with respect to dimensionless time $\Gamma\left(t-t_{0}\right)$, the curves for the different parameters overlap. The case of $\varepsilon_{0}=0$ is special because the rescaling of the energy band on the left lead does not move the resonance from its initial position owing to the extra symmetry. In this case no electron current flows through the device but heat is nevertheless transferred in agreement with the LB prediction. In the inset of panel b) in Fig. 5 we show that the rescaled heat currents for the two different values of $\Gamma$ in the $\varepsilon_{0}=0$ case overlap with each other but not with those with different values of $\varepsilon_{0}$. The current reaches reaches its steady state value in a much shorter time. This might be because the rescaling of the energy band only stretches the resonance width but does not move its position in the special case of $\varepsilon_{0}=0$.

\section{Double quantum dot device}

Lastly, we present the results for the double quantum dot system. The onsite energy of the first quantum dot was set to $\varepsilon_{1}=0$ and the energy difference to the second site $\Delta \varepsilon=\varepsilon_{2}-\varepsilon_{1}$ varied. We present only the results in the reflectionless limit, with $j_{0}=20$. The hopping scaling factor is again $T_{L} / T_{0}=2$. The time evolutions of the current spectral functions for $\Delta \varepsilon=0.2$ are presented in Fig. 6. The current spectral functions now feature two resonances at the respective onsite energies of the two quantum dots. Again we see the effect of the Luttinger field as a stretching of the current spectral function in the left lead. The time dependent electron and heat currents are depicted in Fig. 7. The asymptotic values of the currents coincide with the LB values. The relaxation times are, again, determined by the resonance width $\Gamma$. We see that in contrast to the single quantum dot system the currents exhibit damped oscillations. The frequency of the oscillations is controlled by the difference of the two onsite energies, while the damping and the amplitude are controlled by $\Gamma$. As shown in Refs. [17-19] the presence of multiple bound states in a system may give rise to undamped current oscillations. In our case there are no bound states in the system but the resonances can be made very narrow by decreasing the parameter $\Gamma$. We 

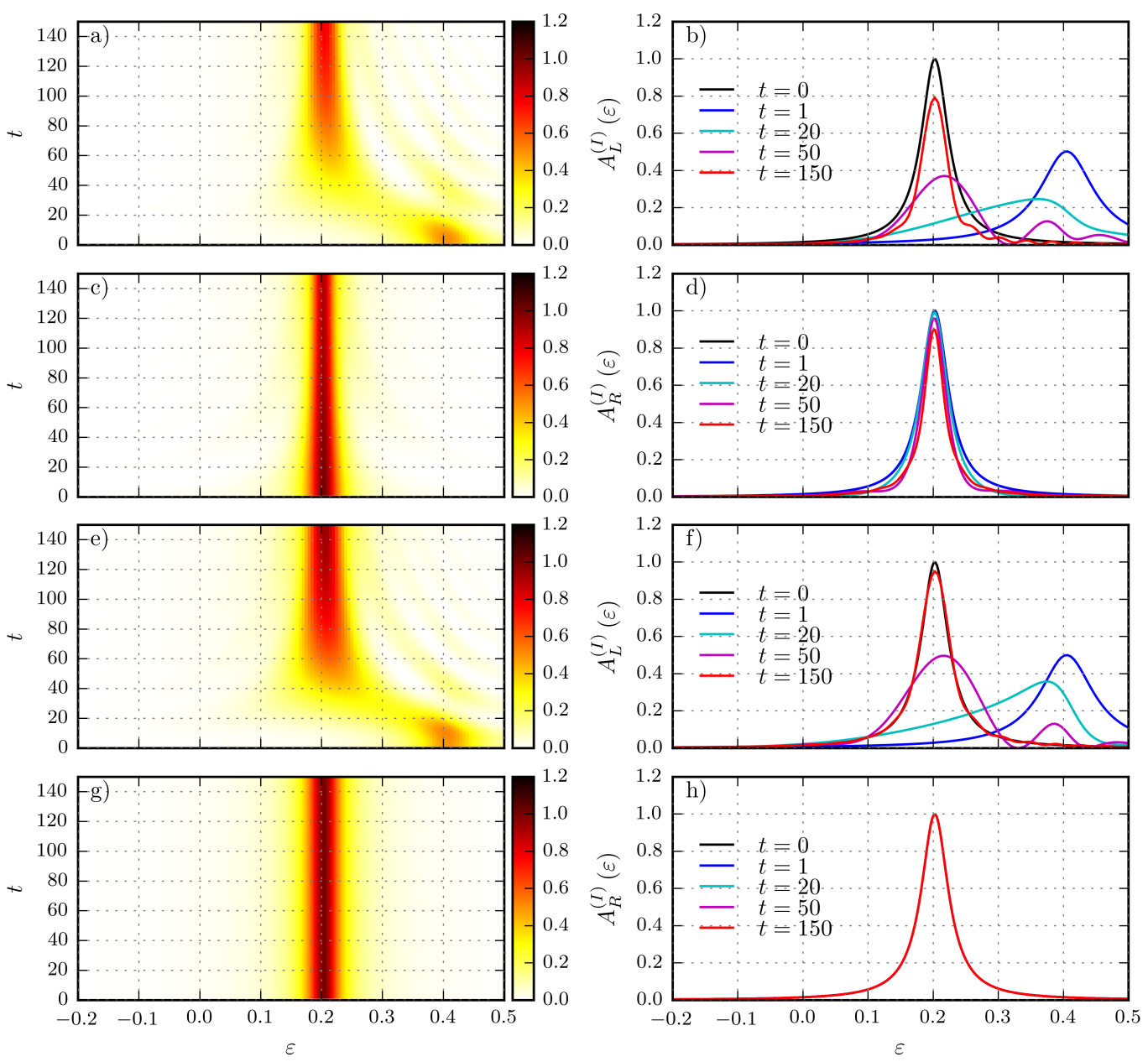

Figure 4. Current spectral functions for the current into the right lead in the single quantum dot device, a) for the left lead $A_{L}^{(I)}(\varepsilon)$ and c) for the right lead $A_{R}^{(I)}(\varepsilon)$ as a function of time in the discontinuous case $j_{0}=0$. b) and d) show the current spectral functions at various times. e), f) show the same quantities as a), b) and g), h) the same as c), d) in the case of the reflectionless limit $j_{0}=20$. The other device parameters are $\varepsilon_{0}=0.2, \Gamma=0.05, T_{0}=0.1, T_{L}=0.2$.

would expect to see the current oscillations to persist for longer times when the resonance is narrowed. Clear indications of this may be seen in both the electron and heat currents depicted in Fig. 7. When the resonance width is changed from $\Gamma=0.05$ (dashed lines) to $\Gamma=$ 0.025 (full lines) additional oscillations occur. This can be most clearly seen in the case where $\Delta \varepsilon=0.4$ (red lines).

\section{CONCLUSION}

The Luttinger field approach proposed by Eich et al. Ref. [11] provides us with a framework in which to simulate time-dependent temperature gradients in nanoscale systems for the purposes of transport calculations. We have seen that a straightforward application of a uniform Luttinger field may produce values of the steady-state heat and electron currents different from those predicted by the LB formula, as well as reverse currents at short times. We have traced the origin of these effects to the scattering occurring because of the discontinuous transition in the energy bands.

Our proposed solution is to apply the Luttinger field in such a way as to introduce a transition region where the hopping parameter of the lead is smoothly varied back to the value it had before the simulated temperature change occurred, right before the lead contacts the scattering region of the device. If the transition region is long enough no additional scattering occurs at the junction between the lead and the scattering region. This was demonstrated in the case of a device consisting of only a lead with no scattering region.

We further applied our reflectionless Luttinger field approach to two sample devices: a single quantum dot device, and a parallel double quantum dot device. In both cases we demonstrated that using this aproach the cor- 

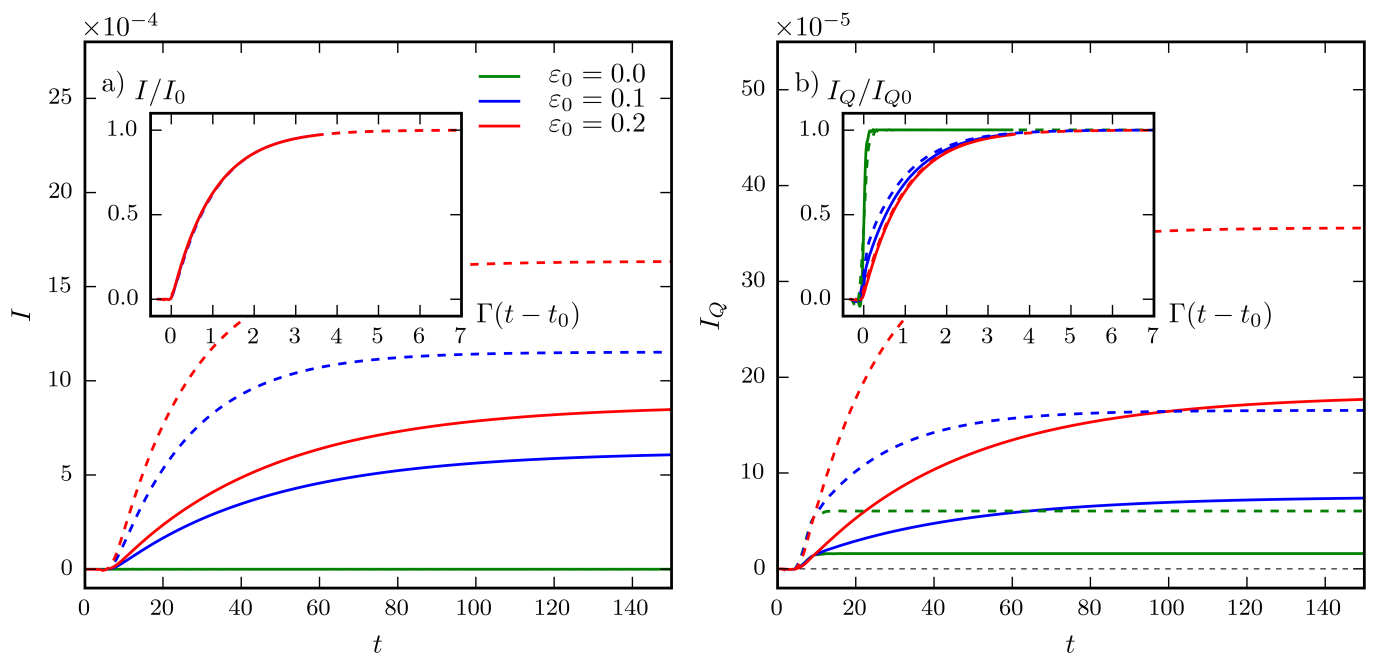

Figure 5. The time dependence of a) the electron current and b) the heat current from the quantum dot into the right lead in the reflectionless limit for different quantum dot energy levels. The temperatures are $T_{0}=0.1, T_{L}=0.2$. The full lines portray the currents at $\Gamma=0.025$ and the dashed lines at $\Gamma=0.05$. The insets show the currents rescaled by the LB values $I_{0}$ and $I_{Q 0}$ with respect to dimensionless time $\Gamma\left(t-t_{0}\right)$, where the initial time delay incurred because of the transition region is estimated to $t_{0}=7$.
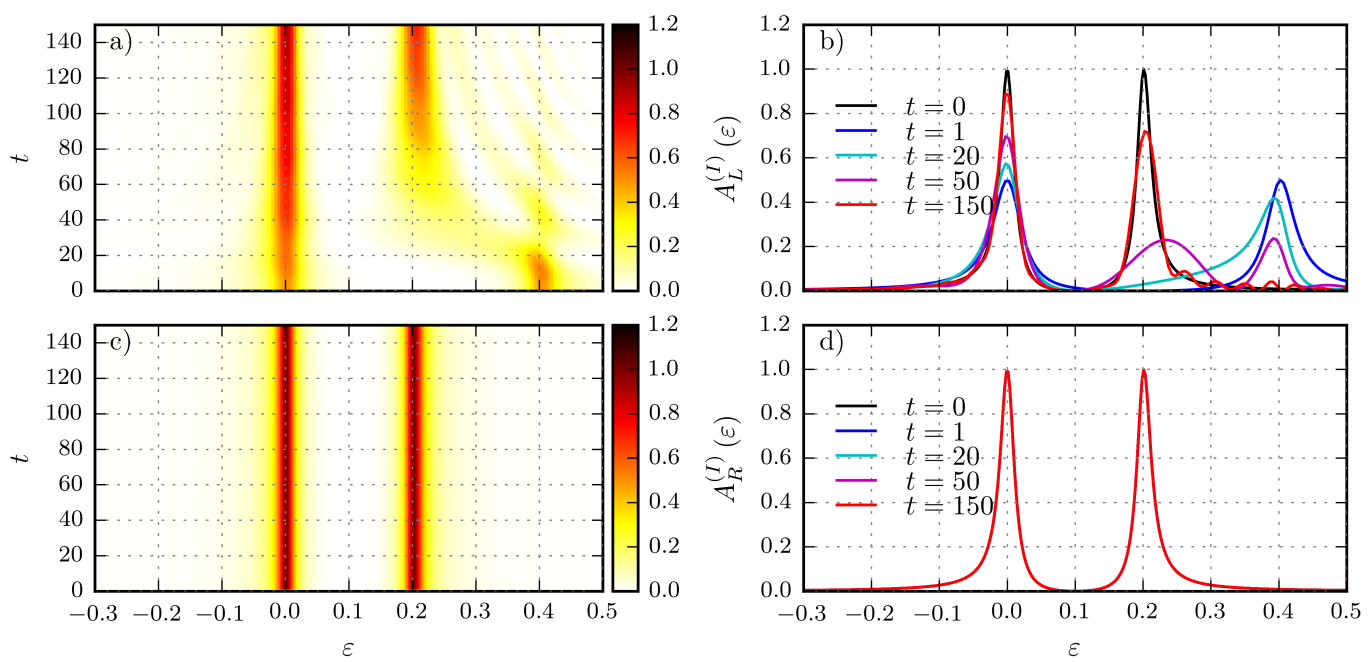

Figure 6. Current spectral functions for the current into the right lead in the double quantum dot device, a) for the left lead $A_{L}^{(I)}(\varepsilon)$ and c) the right lead $A_{R}^{(I)}(\varepsilon)$ as a function of time in the reflectionless limit $j_{0}=20$. b) and d) show the current spectral functions at various times. The other device parameters are $\varepsilon_{0}=0, \Delta \varepsilon=0.2, \Gamma=0.025, T_{0}=0.05, T_{L}=0.1$.

rect LB stationary currents are produced and that the initial reverse current vanished in the case of the single quantum dot.

There is a time delay between the moment the Luttinger field is switched on and the time the correct current spectral function is established at the end of the lead. This delay, being of the order of the inverse of the bandwidth in the lead, is typically short compared to the characteristic time of the nanodevice and is thus of little consequence to the transient response.

As a byproduct of this study we generalized the scheme for time propagation of single electron states under the influence of external bias by Kurth et al. to include the effects due to switching on the Luttinger field. This allows one to numerically model systems where both the bias and the temperature in the leads may vary in time.

In this work we considered only the case where the temperature in a lead is increased. The same approach could also be used when the temperature is decreases but unfortunately strong oscillation of the currents occur in this case due to electrons originating from the opposite lead being reflected by the Luttinger field profile. A way 

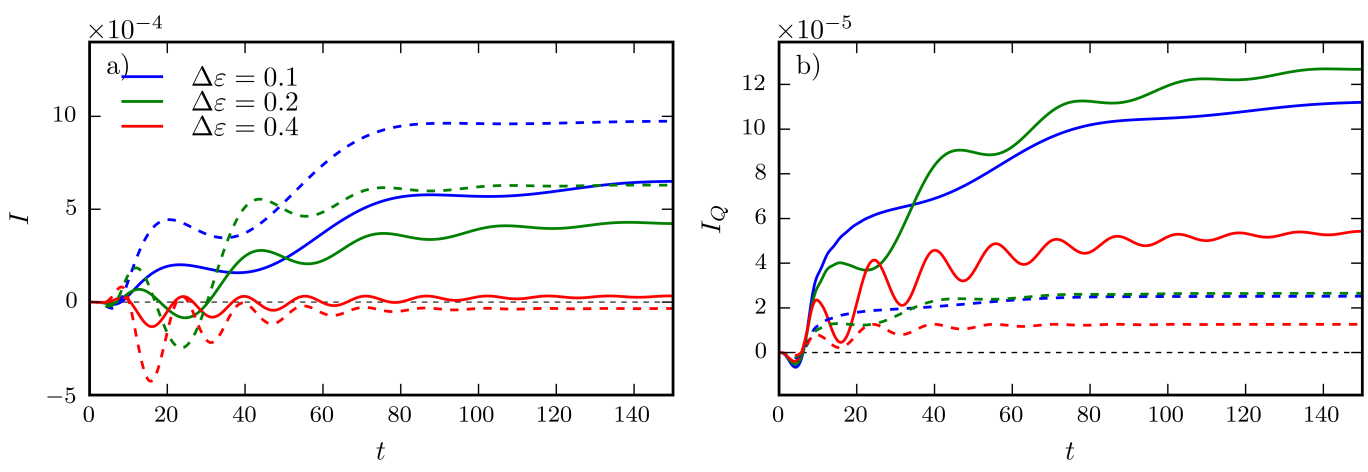

Figure 7. The time dependence of a) the electron current and b) the heat current from the double quantum dot device into the right lead in the reflectionless limit $j_{0}=20$. The energy of the first dot is fixed to $\varepsilon_{0}=0$ and the energy of the second dot varied. The full lines depict the currents at $\Gamma=0.025$ and the dashed lines at $\Gamma=0.05$. The temperatures are $T_{0}=0.05$, $T_{L}=0.1$.

to circumvent this problem would be to start with the reflectionless Luttinger field on and switching it off at the time the temperature is decreased. Initially, the left lead together with the nanodevice should be influenced by the full value of the Luttinger field while the transition region should be positioned at the beginning of the right lead.

Our method, reproducing the correct couplings of the nanodevice to the leads after the Luttinger field is switched on, would be particularly suited to the study of systems where no steady state is established in the long time limit where persistent current oscillations are present.

\section{ACKNOWLEDGMENTS}

C. L. acknowledges the financial support from the Slovenian Research Agency (research core funding No. P1-0306). T. R. acknowledges the financial support from the Slovenian Research Agency (research core funding No. P1-0044).

\section{Appendix A: Numerical method}

To solve the time dependent Schrödinger equation

$$
i \frac{\partial}{\partial t} \psi(t)=H(t) \psi(t)
$$

where the initial wavefunction $\psi(t=0)$ is a scattering state with energy $\varepsilon$ originating from lead $\alpha$, we adapted the scheme proposed by Kurth et al. in Ref. [4]. Namely, to propagate the wavefunction from time $t$ to time $t+\Delta t$ we use

$$
\psi(t+\Delta t)=\frac{1-i \delta H(t+\delta)}{1+i \delta H(t+\delta)} \psi(t)
$$

where $\delta=\Delta t / 2$. Such a propagator is unitary and accurate to the second order in $\Delta t$.

The system is divided into a central region $C$ and leads $\alpha$, i.e.

$$
H(t)=\left(\begin{array}{cc}
H_{C}(t) & H_{C \alpha}(t) \\
H_{\alpha C}(t) & H_{\alpha}(t)
\end{array}\right)
$$

and

$$
\psi(t)=\left(\begin{array}{l}
\psi_{C}(t) \\
\psi_{\alpha}(t)
\end{array}\right)
$$

Here, in contrast to notation in the main text, the region $C$ contains both the scattering region and the transition regions in the leads. The leads defined this way are translationally invariant.

Taking into account the division of the system Eq. (A1) reads

$$
\begin{gathered}
\left(1+i \delta H_{C}^{(n)}\right) \psi_{C}^{(n+1)}=\left(1-i \delta H_{C}^{(n)}\right) \psi_{C}^{(n)}+\sum_{\alpha}\left(-i \delta H_{C \alpha}^{(n)}\right)\left[\psi_{\alpha}^{(n)}+\psi_{\alpha}^{(n+1)}\right] \\
\left(1+i \delta H_{\alpha}^{(n)}\right) \psi_{\alpha}^{(n+1)}=\left(1-i \delta H_{\alpha}^{(n)}\right) \psi_{\alpha}^{(n)}+\left(-i \delta H_{\alpha C}^{(n)}\right)\left[\psi_{C}^{(n)}+\psi_{C}^{(n+1)}\right]
\end{gathered}
$$

where we introduced $H^{(n)}=H(n \Delta t+\delta)$ and $\psi^{(n)}=\psi(n \Delta t)$. We are only interested in the explicit solution for the wave function of the central region $\psi_{C}(t)$ and thus wish to express the lead wave functions $\psi_{\alpha}(t)$ in terms of $\psi_{C}(t)$. 
We introduce the Green's function $G_{\alpha}\left(t, t^{\prime}\right)$ for lead $\alpha$ :

$$
\left(1+i \delta H_{\alpha}^{(n)}\right) G_{\alpha}^{(n+1, m)}=\left(1-i \delta H_{\alpha}^{(n)}\right) G_{\alpha}^{(n, m)}+\delta_{n m}
$$

where $G_{\alpha}^{(n, m)}=G_{\alpha}(n \Delta t, m \Delta t)$. A lead consists of unit cells with the Hamiltonian $h_{\alpha}(t)$ and nearest cell coupling $v_{\alpha}(t)$. For the time propagation of $\psi_{C}$ it is sufficient to know the the Green's function of the first site of each lead $g_{\alpha}^{(n, m)}$. This is calculated from

$$
\left(1+i \delta h_{\alpha}^{(n)}+\delta^{2} v_{\alpha}^{(n)} g_{\alpha}^{(n+1, n)} v_{\alpha}^{\dagger(n)}\right) g_{\alpha}^{(n+1, n)}=1
$$

and

$$
\begin{gathered}
\left(1+i \delta h_{\alpha}^{(n)}+\delta^{2} v_{\alpha}^{(n)} g_{\alpha}^{(n+1, n)} v_{\alpha}^{\dagger(n)}\right) g_{\alpha}^{(n+1, m)}+\delta^{2} v_{\alpha}^{(n)} g_{\alpha}^{(n+1, m)} v_{\alpha}^{\dagger(m)} g_{\alpha}^{(m+1, m)}= \\
=\left(1-i \delta h_{\alpha}^{(n)}-\delta^{2} v_{\alpha}^{(n)} g_{\alpha}^{(n+1, n)} v_{\alpha}^{\dagger(n)}\right) g_{\alpha}^{(n, m)}-\delta^{2} v_{\alpha}^{(n)} g_{\alpha}^{(n, m)} v_{\alpha}^{\dagger(m)} g_{\alpha}^{(m+1, m)}- \\
\quad-\delta^{2} v^{(n)} \sum_{n^{\prime}=m+1}^{n-1}\left(g_{\alpha}^{\left(n+1, n^{\prime}\right)}+g_{\alpha}^{\left(n, n^{\prime}\right)}\right) v_{\alpha}^{\dagger\left(n^{\prime}\right)}\left(g_{\alpha}^{\left(n^{\prime}+1, m\right)}+g_{\alpha}^{\left(n^{\prime}, m\right)}\right) .
\end{gathered}
$$

Using the Green's function of the first site of the lead, the lead's wave function can now be expressed in terms of the central region's wave function,

$$
\psi_{\alpha}^{(n)}=\phi_{\alpha}^{(n)}+\sum_{m=0}^{n-1} g_{\alpha}^{(n, m)}\left(-i \delta H_{\alpha C}^{(m)}\right)\left[\psi_{C}^{(m)}+\psi_{C}^{(m+1)}\right]
$$

where $\phi_{\alpha}(t)$ is a solution for an isolated lead. At $t=0$ the wave function on site $i$ of the lead $\alpha$ can be expanded in terms of plane waves as

$$
\phi_{\alpha i}^{(0)}=\sum_{p} \lambda_{\alpha p} c_{\alpha p}^{(0)} e^{i k_{\alpha p} i}
$$

where the wavevectors $k_{\alpha p}$ and expansion coefficients $c_{\alpha p}^{(0)}$ are solutions of a quadratic eigenvalue problem

$$
\left(v_{\alpha}^{(0) \dagger} e^{-i k_{\alpha}}+h_{\alpha}^{(0)}+v_{\alpha}^{(0)} e^{i k_{\alpha}}\right) c_{\alpha}^{(0)}=\varepsilon c_{\alpha}^{(0)} .
$$

Finally, the central region's wave function is propagated
At later times

$$
\phi_{\alpha i}^{(n)}=\sum_{p} \lambda_{\alpha p} c_{\alpha p}^{(n)} e^{i k_{\alpha p} i}+\chi_{\alpha i}^{(n)},
$$

where the index $p$ counts all solutions for the wavevectors over all possible transport modes of lead $\alpha$. The time evolution of $c_{\alpha p}(t)$ is calculated from

$$
\begin{aligned}
& \left(1+i \delta\left(v_{\alpha}^{(n) \dagger} e^{-i k_{\alpha p}}+h_{\alpha}^{(n)}+v_{\alpha}^{(n)} e^{i k_{\alpha p}}\right)\right) c_{\alpha p}^{(n+1)}= \\
& =\left(1-i \delta\left(v_{\alpha}^{(n) \dagger} e^{-i k_{\alpha p}}+h_{\alpha}^{(n)}+v_{\alpha}^{(n)} e^{i k_{\alpha p}}\right)\right) c_{\alpha p}^{(n)}
\end{aligned}
$$

Again, we only need the solution for the first site of the lead $i=0$ and the time evolution for $\chi_{\alpha 0}(t)$ from

$$
\begin{gathered}
\left(1+i \delta h_{\alpha}^{(n)}+\delta^{2} v_{\alpha}^{(n)} g_{\alpha}^{(n+1, n)} v_{\alpha}^{(n) \dagger}\right) \chi_{\alpha 0}^{(n+1)}= \\
=\left(1-i \delta h_{\alpha}^{(n)}-\delta^{2} v_{\alpha}^{(n)} g_{\alpha}^{(n+1, n)} v_{\alpha}^{(n) \dagger}\right) \chi_{\alpha 0}^{(n)}- \\
-\delta^{2} v_{\alpha}^{(n)} \sum_{n^{\prime}=0}^{n-1}\left(g_{\alpha}^{\left(n+1, n^{\prime}\right)}+g_{\alpha}^{\left(n, n^{\prime}\right)}\right) v_{\alpha}^{\left(n^{\prime}\right) \dagger} \chi_{\alpha 0}^{\left(n^{\prime}\right)}+ \\
+i \delta v_{\alpha}^{(n) \dagger} \sum_{p} \lambda_{\alpha p}\left(c_{\alpha p}^{(n+1)}+c_{\alpha p}^{(n)}\right)
\end{gathered}
$$

with $\chi_{\alpha 0}(t=0)=0$. 


$$
\begin{gathered}
{\left[1+i \delta H_{C}^{(n)}-\sum_{\alpha}\left(-i \delta H_{C \alpha}^{(n)}\right) g_{\alpha}^{(n+1, n)}\left(-i \delta H_{\alpha C}^{(n)}\right)\right] \psi_{C}^{(n+1)}=} \\
=\left[1-i \delta H_{C}^{(n)}+\sum_{\alpha}\left(-i \delta H_{C \alpha}^{(n)}\right) g_{\alpha}^{(n+1, n)}\left(-i \delta H_{\alpha C}^{(n)}\right)\right] \psi_{C}^{(n)}+ \\
+\sum_{\alpha}\left(-i \delta H_{C \alpha}^{(n)}\right)\left[\phi_{\alpha 0}^{(n)}+\phi_{\alpha 0}^{(n+1)}\right]+\sum_{\alpha} \sum_{n^{\prime}=0}^{n-1}\left(-i \delta H_{C \alpha}^{(n)}\right)\left(g_{\alpha}^{\left(n+1, n^{\prime}\right)}+g_{\alpha}^{\left(n, n^{\prime}\right)}\right)\left(-i \delta H_{\alpha C}^{\left(n^{\prime}\right)}\right)\left(\psi_{C}^{\left(n^{\prime}\right)}+\psi_{C}^{\left(n^{\prime}+1\right)}\right),
\end{gathered}
$$

using the Green's functions on the first sites of the leads from Eqs. (A2) and (A3) and the solutions from Eq. (A4) for $i=0$.

\section{Appendix B: Eigenstates in the transition region}

In this Appendix we find approximate eigenstates of the Schrödinger equation for a lead,

$$
-\gamma_{j-1, j} \psi_{j-1}-\gamma_{j+1, j} \psi_{j+1}=\varepsilon \psi_{j},
$$

assuming the transition region in the lead is long, i.e. the hopping parameter varies smoothly along the lead. We use a WKB-like ansatz for a right moving (upper sign) and a left moving (lower sign) wavefunction

$$
\psi_{j}=A_{j} e^{ \pm i\left(\frac{1}{2} k_{0}+k_{1}+\ldots+k_{j-1}+\frac{1}{2} k_{j}\right)} .
$$

Taylor expanding to the first order the hopping parameter, $\gamma_{j \pm 1, j}=\gamma_{j} \pm \frac{1}{2} \gamma_{j}^{\prime}$, the amplitude of the wavefunction, $A_{j \pm 1}=A_{j} \pm A_{j}^{\prime}$, and the wavevector, $k_{j \pm 1}=k_{j} \pm k_{j}^{\prime}$, and collecting the terms of the same order of magnitude in the Schrödinger equation we obtain: a) in the zeroth order the local dispersion relation,

$$
\varepsilon=-2 \gamma_{j} \cos k_{j}
$$

and b) in the first order the relation between the amplitude of the wavefunction and the local velocity, $v_{j}=$ $2 \gamma_{j} \sin k_{j}$ :

$$
A_{j} v_{j}^{\prime}+2 A_{j}^{\prime} v_{j}=0 .
$$

Solving for the amplitude of the wavefunction yields

$$
A_{j}=A_{0} \sqrt{\frac{v_{0}}{v_{j}}} .
$$

The presence of such an reflectionless transition region does not lead to scattering, provided the eigenenergy lies inside the local energy band, $|\varepsilon|<2 \gamma_{j}$, at each point along the lead. To the leading order, the current carried by such a state is

$$
j_{j+1, j}=2 \gamma_{j+1, j} \operatorname{Im}\left\{\psi_{j}^{*} \psi_{j+1}\right\}= \pm\left|A_{j}\right|^{2} v_{j}= \pm\left|A_{0}\right|^{2} v_{0} .
$$

The steady-state current spectral functions are obtained by inserting Eq. (B2) into Eq. (4). The local velocity cancels out with $\frac{d k}{d \varepsilon}$. The steady-state current spectral functions are thus uniform.
* tomaz.rejec@ijs.si

1 R. Landauer, IBM Journal of Research and Development 1, 223 (1957).

2 M. Büttiker, Phys. Rev. Lett. 57, 1761 (1986).

3 M. Büttiker, Y. Imry, R. Landauer, and S. Pinhas, Phys. Rev. B 31, 6207 (1985).

4 S. Kurth, G. Stefanucci, C.-O. Almbladh, A. Rubio, and E. K. U. Gross, Phys. Rev. B 72, 035308 (2005).

${ }^{5}$ G. Stefanicci and R. van Leeuwen, Nonequilibrium ManyBody Theory of Quantum Systems (Cambridge University Press, 2013).

${ }^{6}$ Y. Dubi and M. Di Ventra, Rev. Mod. Phys. 83, 131 (2011).

7 J. M. Luttinger, Phys. Rev. 135, A1505 (1964).
8 B. S. Shastry, Reports on Progress in Physics 72, 016501 (2009).

9 F. G. Eich, M. Di Ventra, and G. Vignale, Phys. Rev. Lett. 112, 196401 (2014).

10 F. G. Eich, M. Di Ventra, and G. Vignale, J. Phys.: Condens. Matter 29, 063001 (2017).

11 F. G. Eich, A. Principi, M. Di Ventra, and G. Vignale, Phys. Rev. B 90, 115116 (2014).

12 G. Tatara, Phys. Rev. Lett. 114, 196601 (2015).

13 G. Tatara, Phys. Rev. B 92, 064405 (2015).

14 R. Biele, R. D'Agosta, and A. Rubio, Phys. Rev. Lett. 115, 056801 (2015).

${ }^{15}$ F. G. Eich, M. Di Ventra, and G. Vignale, Phys. Rev. B 93, 134309 (2016). 
${ }^{16}$ F. Covito, F. G. Eich, R. Tuovinen, M. A. Sentef, and A. Rubio, Journal of Chemical Theory and Computation 14, 2495 (2018).

17 G. Stefanucci, Phys. Rev. B 75, 195115 (2007).
18 E. Khosravi, S. Kurth, G. Stefanucci, and E. K. U. Gross, Applied Physics A 93, 355 (2008).

19 E. Khosravi, G. Stefanucci, S. Kurth, and E. Gross, Phys. Chem. Chem. Phys. 11, 4535 (2009). 